\title{
Discovery of Marine Sponge Compound as Promising Inhibitor for Macrophage Infectivity Potentiator (Mip) Protein against Chlamydia pneumoniae
}

\author{
Ramachandran Vijayan ${ }^{1,2}$, Naidu Subbarao², Natesan Manoharan ${ }^{*}$ \\ ${ }^{1}$ Department of Marine Science, Bharathidasan University, Trichy -620024, India. \\ 2 Center for Computational Biology and Bioinformatics, School of Computational and Integrative Sciences, \\ Jawaharlal Nehru University, New Delhi- 110067, India. \\ * Corresponding author. Tel.: +91-8807862249; email: biomano21@yahoo.com \\ Manuscript submitted March 19, 2015; accepted May 20, 2015. \\ doi: 10.17706/ijbbb.2015.5.3.202-210
}

\begin{abstract}
The macrophage infectivity potentiator (Mip) from Chlamydia pneumoniae is a major virulence factor. Mip is necessary for optimal intracellular infection and survival within both amoeba and human macrophages. Legionella and Chlamydia is the causative agent responsible for Legionnaires' disease, Blindness, Sexual Transmitted Disease (STD) and Pneumonia respectively. Mip exhibits peptidyl-prolyl-cis/trans-isomerase (PPIase) activity that is blocked by the immunosuppressant FK506 or rapamycin, the recent significant increase in the number of reported cases of Blindness, STD and Pneumonia. It is necessary to design of new inhibitors directed against Mip proteins of intracellular pathogens. The aim of this study was to construct the 3D model of Chlamydia pneumoniae Mip by homology modeling. Further, pharmacophore model design and molecular docking simulations were carried out to identify Mip inhibitors from a Universal Natural Product Database (UNPD) of over 229358 natural compounds. The virtual screening furnished 2 hit compounds, from which phorbaside $C$ from Marine sponge Phorbas species and emerged as a top candidate. Along with molecular dynamics (MD) simulations which will be carried out to analyze the binding affinity for the screened novel, lead compounds. Lead compounds will be tested for their ability to inhibit the PPIase activity of Mip proteins in vitro and in vivo.
\end{abstract}

Key words: Chlamydia, Legionella, in-silico mutagenesis, rapamycin, marine sponges.

\section{Introduction}

Chlamydia pneumoniae is an obligate intracellular gram negative bacteria that exhibits a unique developmental cycle [1], [2]. It has been recognized as an important respiratory pathogen associated with acute respiratory diseases that includes pneumonia, bronchitis, and pharnygitis [3], [4]. Macrophage infectivity potentiator (Mip) proteins have also been reported as prominent virulence factor in the pathogenic intracellular bacteria, Legionella pneumophila [5], [6], Chlamydia trachomatis [7] and Chlamydia pneumoniae [8], the etiological agents of human Legionnaires' disease and Chlamydiasis, respectively. Mip has showed the evidence of Peptidyl prolyl cis-trans isomerase activity that can be blocked by Rapamycin [9] and FK506 [9]. Natural products (NPs) play an crucial role in drug discovery [10]. Biologically active natural products would provide selective ligands for abnormal-related targets [11], and eventually influence the disease-related pathways and also shift the biological network from abnormal to healthy state. Universal Natural Product Database (UNPD) is the largest non-commercial and freely available database for 
natural products (http://pkuxxj.pku.edu.cn/UNPD). UNPD consists of 2,29,358 natural products from plants, animals and microorganisms. The UNPD database was developed to be a resource of natural products for virtual screening. Persistent infections are of great attention nowadays in health care, as they are difficult to cure with current antibiotics. Our goal is to design new marine drugs to treat the Chlamydial infections, as the lack of 3-dimensional structure available for Chlamydia pneumoniae Mip has been available. In order to help shed light on the complex mechanism of intracellular infection by these pathogens, homology modeling, pharmacophore model development and virtual screening studies were carried out based on drug design approach[12], [13] for the identification of new potential Mip inhibitors.

\section{Materials and Methods}

\subsection{Homology Modeling}

Crystal structure of the Chlamydia pneumoniae Mip is not yet reported. Therefore, Homology modeling is one of the most accurate computational method which is used for structure prediction. The amino acid sequence of Chlamydia pneumoniae Mip was collected from the Uniprot Database (accession code: Q9Z7P3) (http://www.uniprot.org/uniprot/). On PSI-BLAST search, (http://www.ebi.ac.uk/Tools/sss/psiblast/) was performed known 3D structure revealed that the Legionella pneumophila Mip had the highest score in the sequence similarity with Chlamydia species (ssp) Mip, namely 35\% identity. Hence, in this study the X-ray structure of Legionella pneumophila Mip (Protein Data Bank code: 1FD9) was selected as a template to predict the 3D structure of Chlamydia pneumoniae Mip. Sequence alignment was performed with CLUSTALW, using the BLOSUM matrices [14] for scoring the alignment. Pairwise sequence alignment of the target, Chlamydia ssp. with the template, Legionella pneumophila Mip, is a the crucial step in homology modeling. The obtained alignments were checked for insertions and deletions in the conserved regions manually. The resulting alignment were given as an input for the homology modeling software MODELER [15]. This program assigns the atomic co-ordinates to the regions that are structurally aligned with the template, builds intervening loops, optimizes the rotamers of amino acid side chains, and performs an initial energy optimization of the structure. MODELER [15] generates the protein three-dimensional structures by satisfying the spatial restraints imposed by the sequence alignment with the template. The spatial restraints consist of homology derived restraints on the distances and dihedral angles in the target based on its alignment with the template, stereochemical restraints such as bond length and bond angles from CHARMm force field [16] and the statistical preferences for dihedral angles and non-bonded inter-atomic distances retrieved from a representative set of protein structures. The restraints are expressed in terms of probability density functions (PDF). The three-dimensional homology model of the protein is obtained by probability density function with the target variable target function procedure in the cartesian space that employs methods of conjugate gradients and molecular dynamics with simulated annealing [16]. To achieve sufficient conformational sampling of each active site residue, twenty homology models were generated. Hydrogen atoms were added to the homology models. The homology models were energy minimized with CHARMm force field, in a step-wise manner following a standard procedure consists of 500 steps of steepest descent and 1,000 steps of conjugate gradient with a root mean square (rms) gradient of the potential energy of $0.001 \mathrm{kcal} / \mathrm{mol} \AA$ at each step. The stereo-chemical quality of the final refined model was evaluated using PROCHECK [17].

\subsection{Design of a Novel Pharmacophore}

We used LigandScout [18] to design 3D pharmacophore models based on interactions found in Legionella and E. coli Mip in complex with FK506 and rapamycin. This software incorporates a complete definition of three-dimensional chemical features (such as hydrogen bond donors, acceptors, lipophilic areas, positively 
and negatively ionizable chemical groups) that describe the interaction of a bound small organic molecule (ligand) and the surrounding binding site of the macromolecule. These pharmacophores can be overlaid and superimposed using a pattern-matching based alignment algorithm that is solely based on pharmacophoric feature points instead of chemical structure. From such an overlay, shared features can be interpolated to create a so-called "shared-feature pharmacophore" that shares all common interactions of several binding sites/ligands or extended to create a so-called "merged-feature" pharmacophore [18]. Relevant chemical features, two hydrogen bond donor (HBD), two hydrogen bond acceptor (HBA) and negatively charged, ionizable, NI, are mapped onto the bioactive conformation of Legionella and E. coli Mip in complex with FK506 and rapamycin are combined into a pharmacophore. Ligand Scout which used to align structures and pharmacophores by simply adding them to the alignment lane would give a score. Additional hydrophobic features were also considered. We analyzed the interactions in both the complexes and merging the interactions with the six feature hypothesis defined a pharmacophore model. A number of hypotheses were built, integrating different chemical feature combinations and with increasing complexity to enhance selectivity. This defines shape similarity rather than the shape containment, thus hits could have undesirable protrusions into the active site. To avoid the collisions with protein residues, a set of exclusion volume spheres based on surrounding active sire residues were added to the pharmacophore model.

\subsection{Gold Docking}

Docking was performed using GOLD Software (Genetic Optimization Ligand Docking). GOLD uses a genetic algorithm to explore the full range of ligand conformational flexibility with partial flexibility of the protein [19]. The docking procedure consisted of three interrelated components; a) identification of the binding site b) a search algorithm to effectively sample the search space (the set of possible ligand positions and conformations on the protein surface) and c) a scoring function. The GOLD fitness function consisted of four components: a) protein-ligand hydrogen bond energy (external H-bond); b) protein-ligand van der waals (vdw) energy (external vdw); c) ligand internal vdw energy (internal vdw); d) ligand torsional strain energy (internal torsion). Default settings for GOLD docking were adopted [19].

\section{Results and Discussion}

\subsection{Protein Structure Prediction of $C$. pneumoniae Mip}

Legionella pneumopila Mip consists of two domains namely, $\mathrm{N}$ and C-terminal. $\mathrm{N}$-terminal is responsible for dimerization and C-terminal has a 100 residue domain with 35\% sequence identity to human FKBP12 [20]. Lp Mip exhibits peptidyl prolyl cis-trans isomerase (PPIase) activity which can be inhibited by Rapamycin and FK506 [20]. Homologous proteins are also found in other intra cellular pathogens such as Chlamydia trachomatis and Chlamydia pneumoniae[9]. In the absence of crystal structures for Chlamydia pneumoniae, homology modeling has been shown to be a valuable tool for gaining insight into the structure and function of the proteins. The pairwise sequence identities of the template of Legionella pneumophila Mip (1FD9) with the target, Chlamydia pneumoniae Mip sequence were relatively low, and their similarity were quite high (50\%). Most of the active-site residues were highly conserved. The X-ray crystal structure of the Legionella pneumophila Mip provided a better template for the construction of homology models. Based on the template structure of Legionella Mip, the homology models of Chlamydia pneumoniae Mip was established using MODELER software. The input parameters of the modeling program were set to generate 20 different homology models. Among the 20 models, the ones show the lowest energy, restraint violations, and the conformations of the main chain and side chain were selected for further refinement. The quality of the initial model was further improved by energy minimization as described in the materials and methods section. The energy minimizations may help to remove any steric clashes or improper geometries in the 
protein structure to obtain a model with proper bond angles and bond length.

\subsection{Protein Structure Validation}

In order to check the standard of the homology model of Chlamydia ssp. Mip was validated by PROCHECK program. Red color shows the allowed regions, i.e., right conformation of the amino acid residues. Yellow color regions shows additional allowed regions. White color portion shows the disallowed conformation of the amino acid in the protein structure. Ramachandran plot shows the distribution of the main chain torsion angles, the phi/psi angles of $81.7 \%$ residues fell in the most favored regions, $15.2 \%$ of the residues lay in the additional allowed regions, and 3.1\% residues fell in the generously allowed regions; no residues lay in the disallowed conformations (Fig. 1). The overall conformation of the Chlamydia pneumoniae Mip model was very similar to the template (Fig. 2). Thus, the three-dimensional model of Chlamydia pneumoniae Mip was considered to be reasonable.

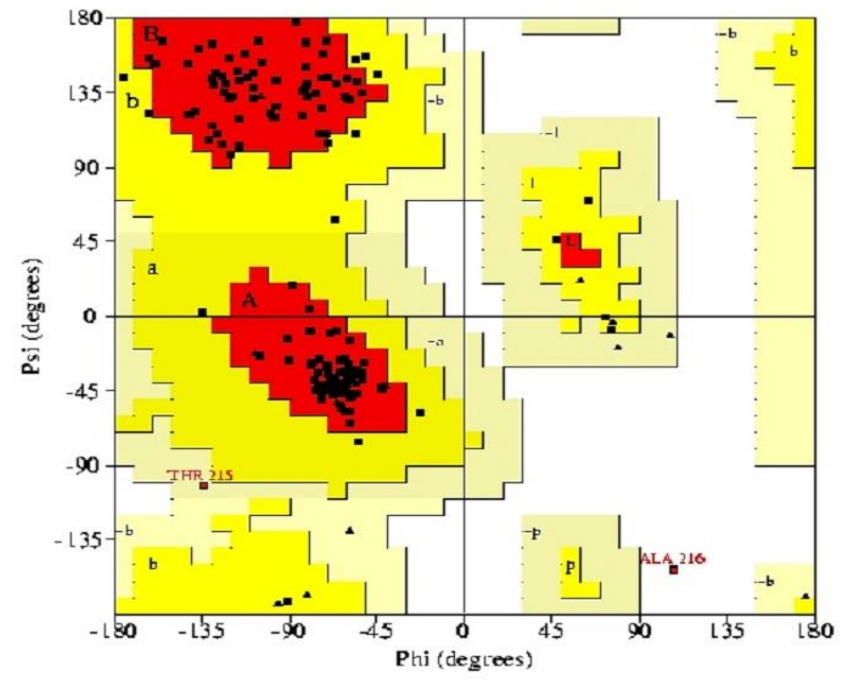

Fig. 1. Ramachandran plot of Chlamydia pneumoniae Mip.

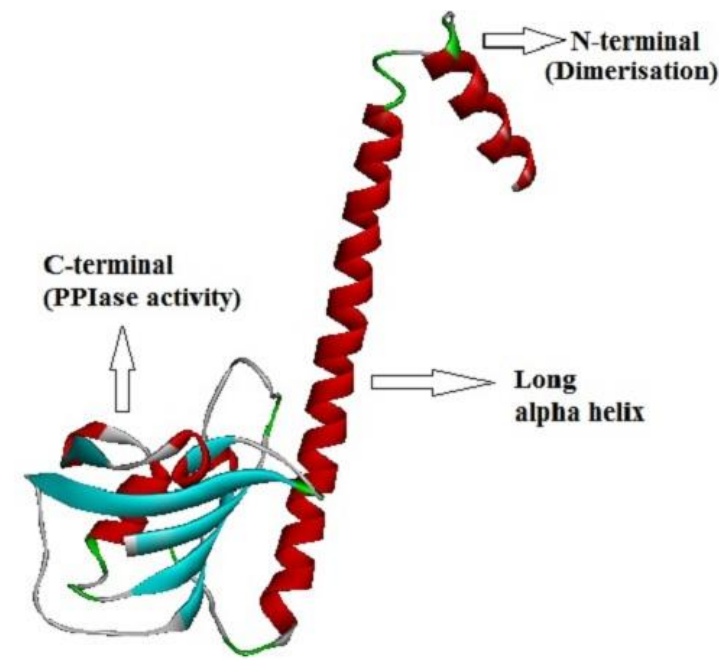

Fig. 2. Cartoon representation of Chlamydia pneumoniae Mip, showing the two domains (N-terminal domain and C-terminal domain). 


\subsection{Pharmacophore Modeling}

In the absence of Chlamydia Mip inhibitors, rapamycin was used as the template for ligand-based pharmacophore modeling. The structure based approach was applied based on the X-ray crystal structure of Legionella and E. coli Mip in complex with FK506 and rapamycin. The active site was carefully analyzed for protein-ligand interactions (Fig. 3). The binding site is characterized by several hydrogen bond interactions and hydrophobic interactions which are also considered as described in materials and methods. Ligand Scout, used to align structures and pharmacophores by superimposing them to the alignment lane, it would give a score. Additional hydrophobic features were also considered. We analyzed the interactions in both the Legionella and E. coli Mip complexes and merging the interactions with the six feature hypothesis defined a pharmacophore model.

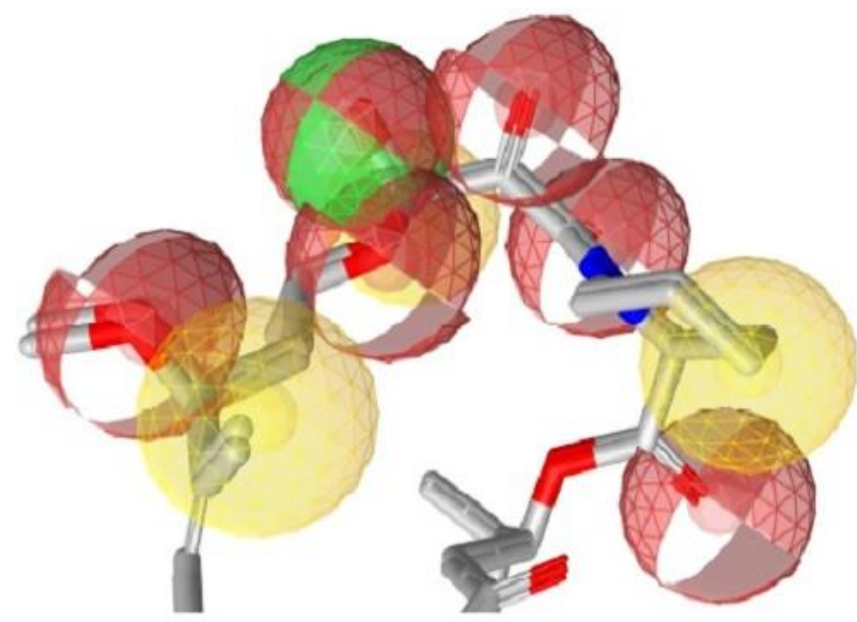

Fig. 3. Two-dimensional structure of the pharmacophore model of rapamycin.

(HBA, hydrogen bond acceptor: green; HBA, hydrogen bond donor: red)

\subsection{Virtual Screening}

In order to find possible new drug candidates targeting Mip proteins, we used the Universal Natural Products Database of Peking University (http://pkuxxj.pku.edu.cn/UNPD) of commercially available compounds (229358 compounds) as a basis for virtual screening. A subset of the database filtered according to the Lipinsky rule and ADME (Absorption, Digestion, Metabolism and Excretion) properties reduced the number to 87,920 compounds (Fig. 4). Applying our pharmacophore model, we were able to rank all compounds in this subset according to their ability to form crucial interactions with Mip. GOLD was used to dock the top 500 compounds after pharmacophore filtering. The crystal structure of Legionella Mip as well as our constructed homology model Chlamydia pneumoniae Mip were used as receptors for the docking. Interestingly, 3 compounds (phorbaside $\mathrm{C}$ from Marine sponge Phorbas species and a novel compound) were found to be strong binding affinity among the top 50 docking solution in Legionella Mip and the homology model of Chlamydia pneumoniae.

\subsection{Hydrogen Bonding Interactions}

The docking studies were performed using the GOLD software [19]. The amino acid residues ASP142 and TYR185 around 5Å were assigned as catalytic region for docking (Fig. 5). The docked complex revealed significant notable features. Calculation of binding energy is very important for understanding the affinity level of biological partners [12], [13]. 


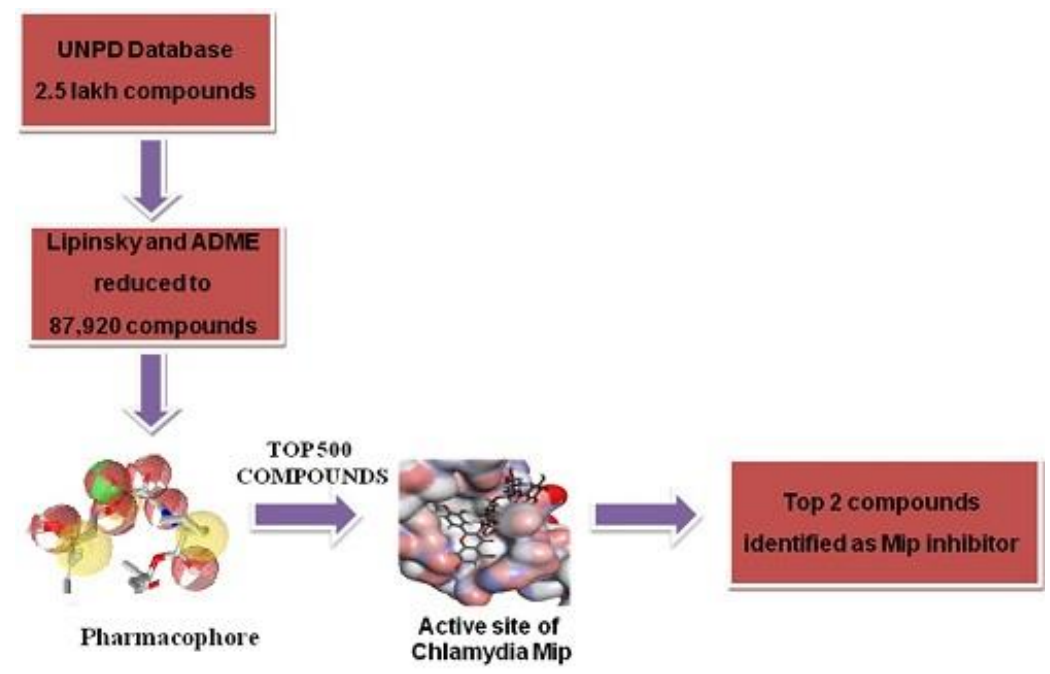

Fig. 4. A schematic work-flow diagram describes the discovery of Mip inhibitors.

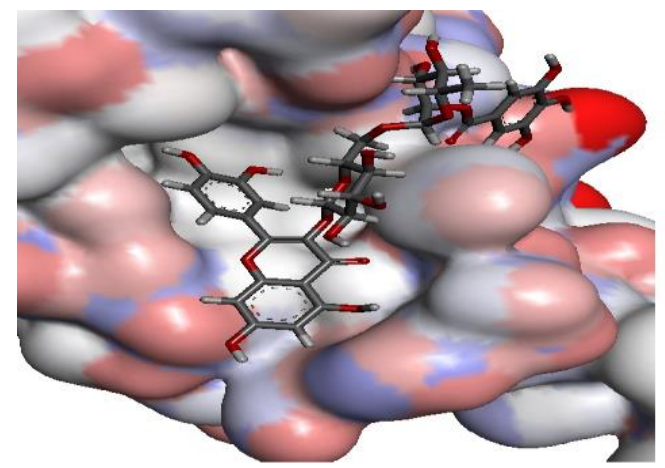

Fig. 5. Molecular docking of novel-compound into the binding site of Chlamydia pneumoniae Mip, having the binding affinity of 70 (gold score).

Table 1. Chemical Descriptors of Marine Sponge Compound Phorbaside C and Novel Compound Discovered from Structure Based Virtual Screening

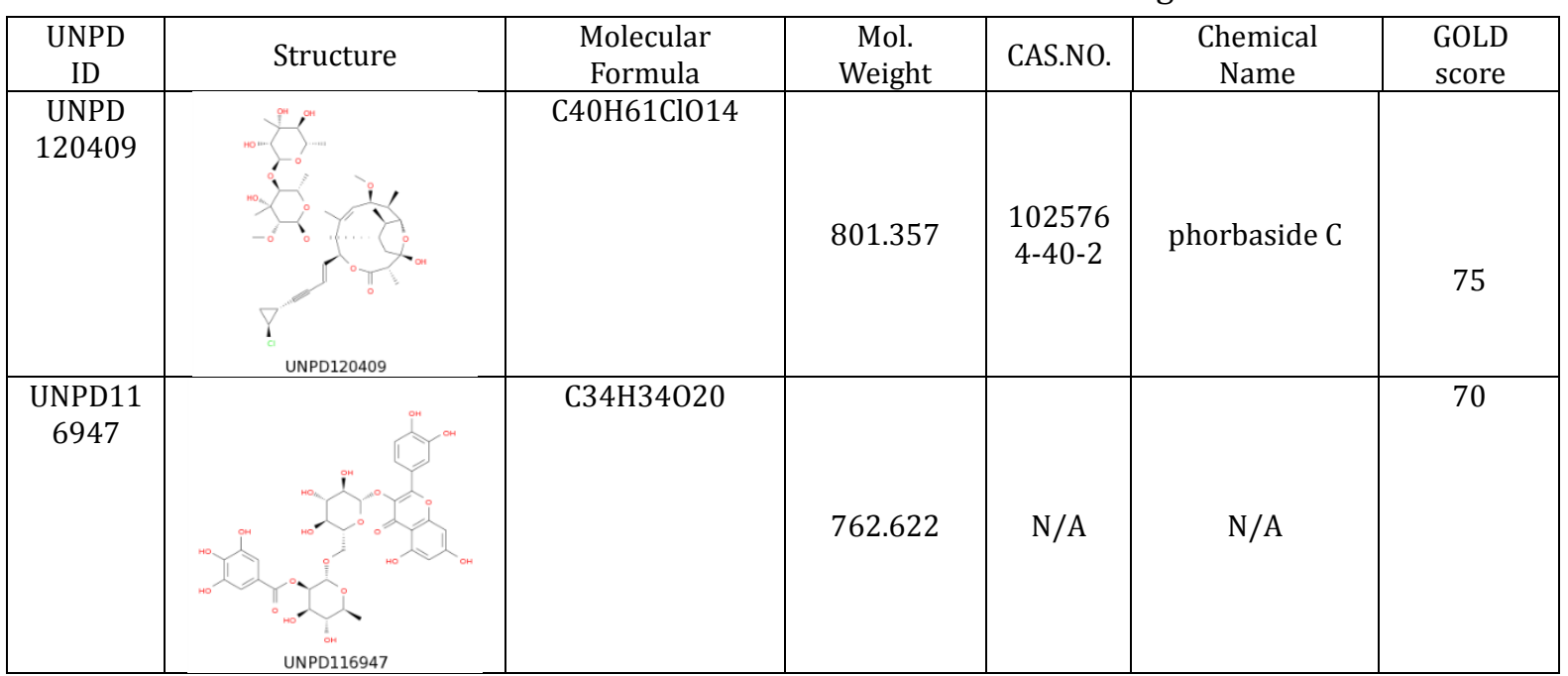

Overall binding energy of the complex mainly contributes to attractive and repulsive vanderWaals interaction energy, electrostatic interactions and hydrogen bond (HB) interactions. The Chlamydia pneumoniae Mip has the binding affinity of 75 with phorbaside $C$ from Marine sponge Phorbas species (Table 1) and (Fig. 6). And the interactions between the novel compound and Chlamydia pneumoniae Mip 
are analyzed and crucial interactions are: Seventeen VanderWaals residues (Leu-157, His-158, Tyr-159, Thr-160, Gly-161, Phe-169, Leu-181, Val-186, Ile-187, Phe-190, Met-194, Thr-215, Ala-216, Leu-225, Phge-227, Glu-228, Val-229), six electrostatic interactions (His-158, Tyr-159, Asp-170, Lys-185, Tyr-213, Gly-214), and Tyr-159 which makes single hydrogen bond interaction was observed in Chlamydia pneumoniae Mip with the phorbaside C from Marine sponge Phorbas species. The Chlamydia pneumoniae Mip has the binding affinity of 70 with novel compound (Table 1) and (Fig. 7).

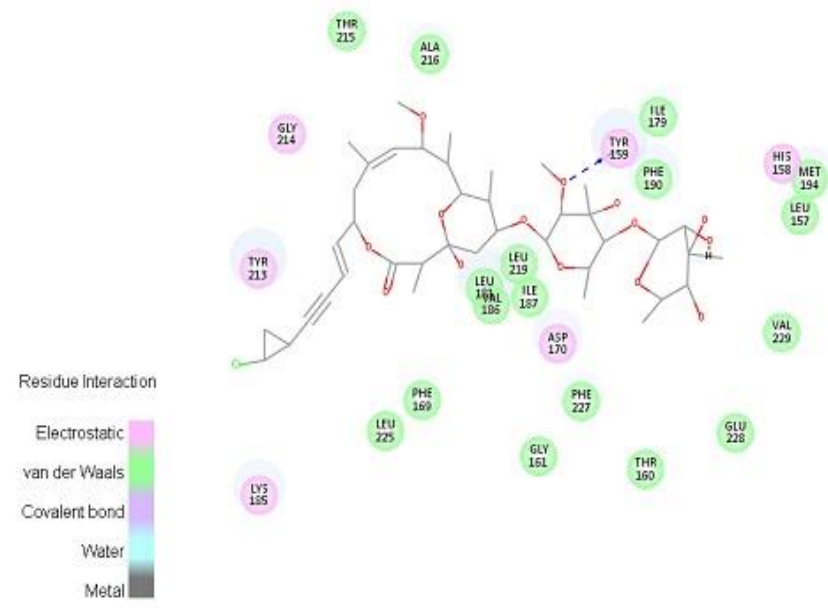

Fig. 6. Hydrogen bond interactions of phorbaside C from Marine sponge Phorbas species with Chlamydia pneumoniae Mip predicted by Discover Studio.

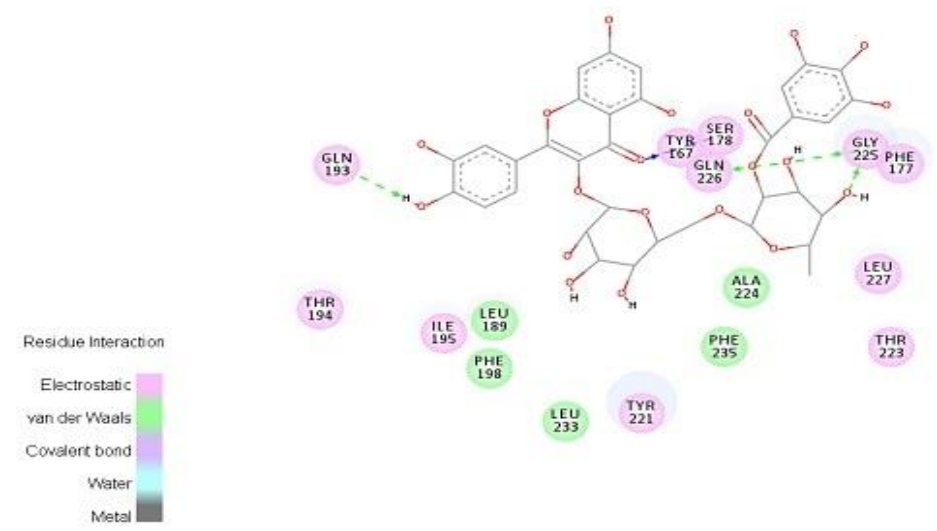

Fig. 7. Hydrogen bond interactions of novel compound with Chlamydia pneumoniae Mip predicted by

Discover Studio

Futher, interactions between the novel compound and Chlamydia pneumoniae Mip were analyzed and crucial interactions are: Five VanderWaals residues(Leu-189, Phe-198, Ala-224, Leu-233, Phe-135), eleven electrostatic interactions(Tyr-167, Phe-177, Ser-178, Gln-193, Thr-194, Ile-195, Tyr-221, Thr-223, Gly-225, Gln-226, Leu-227) and five hydrogen bond interactions(Ser-178, Gln-193, Gly-225, Gly-225, Gln-226). They are observed in Chlamydia pneumoniae Mip with the novel compound.

\section{Conclusion}

In the present work, it indicates that in-silico structure-based virtual screening discovered a natural product as inhibitor for Chlamydia pneumoniae Mip. We validated this approach by designing a pharmacophore model to identify the new potential leads for Chlamydia pneumoniae Mip. Interestingly, 2 out of 50 compounds were found to be the best compound for Chlamydia pneumoniae Mip. Marine sponge 
compound phorbaside $\mathrm{C}$ and a novel compound showed the strong binding affinity and interactions with Chlamydia pneumoniae Mip. Molecular dynamics (MD) simulations will be carried out to analyze the binding affinity for the screened novel lead compounds. Hits found in this study will be tested for PPIase (peptidyl prolyl cis-trans insomerase) inhibition in vitro and for antibacterial effects in vivo.

\section{References}

[1] Grayston, J. T., \& Wang, S. (1975). New knowledge of chlamydiae and the diseases they cause. J. Infect. Dis., 132, 87-105.

[2] Mares, M. (2012). Chlamydia. Rijeka, Croatia: In Tech Janeza Trdine Publishers.

[3] Grayston, J. T., Aldous, M. B., Easton, A., Wang, S. P., Kuo, C. C., Campbell, L. A., et al. (1993). Evidence that Chlamydia pneumoniae causes pneumonia and bronchitis. J. Infect. Dis., 168, 1231-1235.

[4] Allan, R. R., \& Michelle, J. A. (1996). Microbiology of the genitourinary system. In S. Baron, \& T. X. Galveston (Eds.), Medical Microbiology (4th ed.). US: University of Texas Medical Branch, Galveston Publishers.

[5] Fischer, G., Bang, H., Ludwig, B., Mann, K., \& Hacker, J. (1992). Mip protein of Legionella pneumophila exhibits peptidyl-prolyl-cis/trans isomerase (PPlase) activity. Mol. Microbiol., 6(10), 1375-1383.

[6] Steinert, M., Ott, M., LuÄok, P. C., \& Hacker, J. (1994). Studies on the uptake and intracellular replication of Legionella pneumophila in protozoa and in macrophage-like cells. FEMS. Microbiol. Ecol., 15(3-4), 299-308.

[7] Lundemose, A. G., Rouch, D. A., Penn, C. W., \& Pearce, J. H. (1993). The Chlamydia trachomatis Mip-like protein is a lipoprotein. J. Bacteriol., 175(11), 3669-3671.

[8] Essig, A., Heinemann, M., Simnacher, U., \& Marre, R. (1997). Infection of Acanthamoeba castellanii by Chlamydia pneumoniae. Appl. Environ. Microbiol., 63(4), 1396-1399.

[9] Lundemose, A. G., Kay, J. E., \& Pearce, J. H. (1993). Chlamydia trachomatis Mip-like protein has peptidyl-prolyl cis trans isomerase activity that is inhibited by FK506 and rapamycin and is implicated in initiation of Chlamydial infection. Mol. Microbiol., 7, 777-783.

[10] Newman, D. J., \& Cragg, G. M. (2012). Natural products as sources of new drugs over the 30 years from 1981 to 2010. J. Nat. Products, 75(3), 311-335.

[11] Clardy, J., \& Walsh, C. (2004). Lessons from natural molecules. Nature, 432(7019), 829-837.

[12] Vijayan, R., Subbarao, N., \& Mallick, B. N. (2007). In silico modeling of alpha-1 A Adrenoceptor: Interactions of its normal and mutated Active sites with noradrenaline as well as its agonist and antagonist. Am. J. Biochem. Biotech., 3(4), 216-224.

[13] Vijayan, R., Subbarao, N., \& Manoharan, N. (2015). In silico analysis of conformational changes induced by normal and mutation of macrophage infectivity potentiator catalytic residues and its interactions with Rapamycin. Interdiscip. Sciences: Comput Life Sciences, 7, 1-8.

[14] McWilliam, H., Li, W., Uludag, M., Squizzato, S., Park, Y. M., Buso, N., et al. (2013). Analysis tool web services from the EMBL-EBI. Nucleic. Acids. Res., 41, 597-600.

[15] Eswar, N., John, B., Mirkovic, N., Fiser, A., Ilyin, V. A., Pieper, U., et al. (2003). Tools for comparative protein structure modeling and analysis. Nucleic Acids Res., 31(13), 3375-3380.

[16] Brooks, B. R., Bruccoleri, R. E., Olafson, B. D., States, D. J., Swaminathan, S., \& Karplus, M. (1983). CHARMM: A program for macromolecular energy, minimization, and dynamics calculations. J. Comp. Chem., 4(2), 187-217.

[17] Laskowski, R. A., MacArthur, M. W., Moss, D. S., \& Thornton, J. M. (1993). PROCHECK: A program to check the stereochemical quality of protein structures. J. Appl. Cryst., 26(2), 283-291.

[18] Wolber, G., \& Langer, T. (2005). LigandScout: 3-D pharmacophores derived from protein-bound ligands 
and their use as virtual screening filters. J. Chem. Inf. Model, 45(1), 160-169.

[19] Jones, G., Willett, P., Glen, R. C., Leach, A. R., \& Taylor, R. (1997). Development and validation of a genetic algorithm for flexible docking. J. Mol. Biol., 267(3), 727-748.

[20] Riboldi-Tunniclie, A., Konig, B., Jessen, S., Weiss, M. S., Rahfeld, J., Hacker, J., et al. (2001). Crystal structure of MIP, a prolylisomerase from Legionella pneumophila. Nature Struct. Biol., 8(9), 779-783.

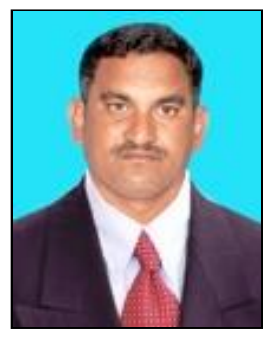

N. Manoharan obtained his $\mathrm{PhD}$ in marine biochemistry and pharmacology from Bharathidasan University. Presently, he is working as an assistant professor in the Department of Marine Science, Bharathidasan University, Trichy, India. His area of research work and specialization focus on marine pharamacology and toxicology.

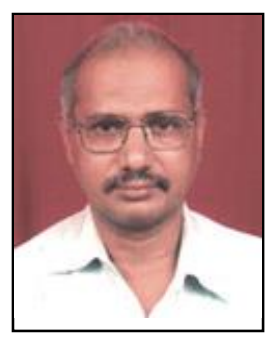

N. Subbarao obtained his MSc and PhD in IIT Kanpur. He received the Common WealthJawaharlal Nehru Centenary Postdoctoral Fellowship and worked as a post doctoral fellow at the Department of Biochemistry and Molecular Biology, University of Leeds, UK in the year 1990-1992. He worked as a junior information scientist at the Bose Institute, Calcutta in the year, 1993-1995. Also he worked as an information scientist / system analyst at the School Computational and Integrative Sciences, Jawaharlal Nehru University during 1995-2013. Presently, he is working as an associate professor in the Center for Computational Biology and Bioinformatics, School of Computational and Integrative Sciences, JNU, Delhi, India. His area of research work and specialization focus on molecular modeling and drug designing, cooperativity in macromolecules. 